\title{
Use of fluorine-18 sodium fluoride for the diagnosis of transthyretin cardiac amyloidosis: The birth of a new technique
}

\author{
Cesia Gallegos, MD, ${ }^{\mathrm{a}}$ and Edward J. Miller, MD, $\mathrm{PhD}^{\mathrm{a}}$ \\ a Section of Cardiovascular Medicine, Yale University School of Medicine, New Haven, CT
}

Received Feb 24, 2017; accepted Feb 24, 2017

doi:10.1007/s12350-017-0847-6

\section{See related article, pp. 1559-1567}

Imaging techniques undergo a process of development. They are birthed from an idea or a hypothesis, and then investigators look for pre-clinical models followed by publication of clinical case reports. In the adolescence of a technique, proof-of-concept series set the stage for larger scale trials of validation. Evidence for utility builds and grows until the technique reaches maturity by demonstration of value to patients and providers. It is within this framework that Mogenstern et al report intriguing data on the early development of ${ }^{18} \mathrm{~F}-\mathrm{NaF}$ imaging for the evaluation of cardiac amyloidosis.

Cardiac amyloidosis (CA) is caused by deposition of insoluble proteins in the heart resulting in hypertrophy, progressing to diastolic dysfunction and heart failure with preserved ejection fraction $(\mathrm{HFpEF}){ }^{1,2}$ It exists in two predominant forms: acquired monoclonal light chain (AL) vs transthyretin-related (ATTR). ATTR-CA is much more prevalent, especially in the elderly, and can be further subclassified as genetically normal (wild type or senile systemic amyloidosis, SSA) or genetically abnormal (mutant type or familial amyloid cardiomyopathy, FAC). Senile systemic amyloidosis increases in prevalence in men older than 60 years of age, and since the clinical presentation of HFpEF may be attributed to hypertension and other causes of diastolic dysfunction that are also common in the aged

Reprint requests: Edward J. Miller, MD PhD, Section of Cardiovascular Medicine, Yale University School of Medicine, 333 Cedar Street, PO Box 208017, New Haven, CT 06520-8017; edward.miller@yale.edu

J Nucl Cardiol 2018;25:1568-70.

$1071-3581 / \$ 34.00$

Copyright (C) 2017 American Society of Nuclear Cardiology. population, the diagnosis of ATTR-CA can be challenging and the prevalence of the disease underappreciated. The distinction between ATTR-CA and AL-CA is of paramount importance as prognosis and therapy vary significantly depending on the subtype.

Despite the growing knowledge of ATTR-CA as a cause of HFpEF, there has been an unmet clinical need to provide better noninvasive methods to diagnose it. Over the last 5-10 years, there has been a dramatic increase in investigations of multimodality imaging to diagnose CA and differentiate AL-CA from ATTR-CA. In particular, nuclear cardiology techniques using boneavid SPECT tracers $\left({ }^{99 \mathrm{~m}}\right.$ technetium pyrophosphate ( ${ }^{99 \mathrm{~m}}$ Tc-PYP), $\quad{ }^{99 m}$ technetium 3, 3-diphosphono-1,2propanodicarboxylic acid ( $\left.{ }^{99 \mathrm{~m}} \mathrm{Tc}-\mathrm{DPD}\right)$, and ${ }^{99 \mathrm{~m}_{\text {tech- }}}$ netium hydroxydiphosphonate $\left.\left({ }^{99 \mathrm{~m}} \mathrm{Tc}-\mathrm{HDP}\right)\right)$ have shown great promise. In 2013, the group responsible for the current manuscript ${ }^{3}$ proposed that ${ }^{99 m}$ Tc-PYP imaging may provide a means to differentiate between ATTR and AL-CA. In 2016, the use of ${ }^{99 m}$ Tc-PYP to diagnose ATTR-CA was further standardized by this group, ${ }^{4}$ and a multi-center consensus statement demonstrated the utility of all the bone-avid SPECT tracers to provide a highly accurate noninvasive differentiation between ATTR and AL-CA. ${ }^{5}$

On this background, the manuscript by Morgenstern et $\mathrm{al}^{6}$ in this issue of Journal of Nuclear Cardiology evaluates a potential other means of distinguishing ATTR-CA from AL-CA, using the PET tracer ${ }^{18}$ fluorine-labeled sodium fluoride $\left({ }^{18} \mathrm{~F}-\mathrm{NaF}\right)$. This tracer is approved by the FDA for prostate cancer screening, and has also been studied for detecting microcalcifications in coronary plaques as well as predicting progression of disease in patients with calcific aortic stenosis. In the current work, the authors present a prospective pilot study in which they enrolled 7 patients with biopsyproven CA ( 2 with AL-CA, 5 with ATTR-CA) who all underwent ${ }^{18} \mathrm{~F}-\mathrm{NaF}$ PET imaging. The control patients $(\mathrm{N}=5)$ were retrospectively assessed, and had 
undergone ${ }^{18} \mathrm{~F}-\mathrm{NaF}$ PET imaging for prostate cancer and had no medical history of amyloidosis. Age and gender of study and control individuals were similar. Scans were assessed qualitatively and quantitatively for uptake of ${ }^{18} \mathrm{~F}-\mathrm{NaF}$. The principal finding of the study was significantly greater ${ }^{18} \mathrm{~F}-\mathrm{NaF}$ PET uptake in patients with cardiac amyloid than in controls (Figure $3 ; P=0.018$ ), with greater uptake in patients with ATTR-CA vs ALCA and controls. Mean SUV of AL-CA and control patients was similar $(P=0.434)$. In addition, there was diffuse uptake of ${ }^{18} \mathrm{~F}-\mathrm{NaF}$ with some prominent, focal myocardial uptake in some segments in the images from ATTR-CA patients.

The results in this study add to the preliminary evidence of ${ }^{18} \mathrm{~F}-\mathrm{NaF}$ as a potential agent in the diagnosis and differentiation between ATTR and AL-CA, based on the hypothesis that the type of amyloid protein affects local calcium homeostasis. Previously, two case reports with discordant results had been published. ${ }^{7,8}$ One report suggested that ${ }^{18} \mathrm{~F}-\mathrm{NaF}$ PET/CT imaging was positive in a patient with mutant (Val122Ile) ATTR-CA but negative in a patient with AL-CA. ${ }^{8}$ A second case report showed no ${ }^{18} \mathrm{~F}-\mathrm{NaF}$ uptake in a patients with WT or Ile68Leu ATTR-CA. However, a recently reported small case series (4 ATTR-CA (2 V122I, 2 WT); 3 AL, 7 controls) demonstrated that hybrid PET/magnetic resonance (MR) using ${ }^{18} \mathrm{~F}-\mathrm{NaF}$ might aid in the differentiation of ATTR and AL-CA, reporting increased patchy uptake in areas of ATTR colocalizing to regions of late gadolinium enhancement, ${ }^{9}$ supporting the conclusions made by Morgenstern et al.

However, despite the promising results, this study does have some limitations that could be answered in future larger studies. First, the small sample size limits the assessment of the potential differences between ATTR-CA and AL-CA. With only two AL-CA patients in the study, it is difficult to understand whether or not ${ }^{18} \mathrm{~F}-\mathrm{NaF}$ can reliably distinguish between these two entities. It should be pointed out that 1 of the 2 patients with AL-CA in the study had normal IVSD (1.0) and LVPW (0.9), suggesting the degree of AL cardiac involvement in this patient was not extreme. Another issue is the control group of patients, which did not have suspected CA and lacked description of morphological features of $\mathrm{CA}$, such as cardiac hypertrophy and/or diastolic heart failure, raising the question whether the observed increase in ${ }^{18} \mathrm{~F}-\mathrm{NaF}$ signal is specific to $\mathrm{CA}$. Also, there is no description of the reproducibility or repetition accuracy of the imaging findings. Lastly, given the tremendous experience in this group with ${ }^{99 m}$ Tc-PYP imaging and the rapid validation of PYP imaging as the 'gold standard' of noninvasively differentiating ATTR-CA from AL-CA, a comparison of ${ }^{18} \mathrm{~F}$ $\mathrm{NaF}$ with ${ }^{99 \mathrm{~m}} \mathrm{Tc}-\mathrm{PYP}$ in patients in this study would have been very useful. With these limitations in mind, the data in this paper are intriguing and should be interpreted as showing that ${ }^{18} \mathrm{~F}-\mathrm{NaF}$ PET has increased signal in patients with CA more than normal controls without known heart disease. The authors should be commended for their novel quantitative approach.

As nuclear cardiology progresses and we move forward in our search for novel tracers, new molecular targets, and imaging protocols beyond traditional myocardial perfusion imaging, we need to ensure the continued iterative advancement that lead to the overwhelming success of our field. In particular, with all new nuclear cardiology techniques, we need to developmentally focus on moving from proof of concept (as Morgenstern et al do in this manuscript) to providing validation, reproducibility, and comparison to 'gold standards.' Studies such as by Morgenstern et al aim to better define disease and expand our imaging armamentarium in cardiac amyloidosis, but ${ }^{18} \mathrm{~F}-\mathrm{NaF}$ imaging for cardiac amyloidosis is still in its infancy. The authors rightly point out that investment in research and development of new imaging techniques to better (and potentially earlier) define cardiac amyloidosis involvement is critically needed. As our fields seek to successfully mature techniques such as ${ }^{18} \mathrm{~F}-\mathrm{NaF}$ PET for cardiac amyloidosis, we need to keep in mind and paraphrase a commercial by Orson Welles for Paul Masson wine that aired in the late 1970's around the time of the birth of nuclear cardiology, "Some things cannot be rushed. We will sell no wine (or new imaging technique) before its time.",

\section{Disclosure}

There is no financial disclosure relevant to this publication. The content is solely the responsibility of the authors.

\section{References}

1. Ruberg FL, Berk JL. Transthyretin (TTR) cardiac amyloidosis. Circulation 2012;126:1286-300. doi:10.1161/CIRCULATIONAHA. 111.078915 .

2. Falk RH, Alexander KM, Liao R, Dorbala S. AL (Light-Chain) cardiac amyloidosis: A review of diagnosis and therapy. J Am Coll Cardiol 2016;68:1323-41. doi:10.1016/j.jacc.2016.06.053.

3. Bokhari S, Castaño A, Pozniakoff T, Deslisle S, Latif F, Maurer MS. (99m)Tc-pyrophosphate scintigraphy for differentiating lightchain cardiac amyloidosis from the transthyretin-related familial and senile cardiac amyloidoses. Circ Cardiovasc Imaging 2013;6:195-201. doi:10.1161/CIRCIMAGING.112.000132.

4. Bokhari S, Morgenstern R, Weinberg R, Kinkhabwala M, Panagiotou D, Castano A, et al. Standardization of 99mTechnetium pyrophosphate imaging methodology to diagnose TTR cardiac amyloidosis. J Nucl Cardiol 2016. doi:10.1007/s12350-016-0610-4. 
5. Gillmore JD, Maurer MS, Falk RH, Merlini G, Damy T, Dispenzieri A, et al. Nonbiopsy diagnosis of cardiac transthyretin amyloidosis. Circulation 2016;133:2404-12. doi:10.1161/CIRCULATIONAHA. 116.021612 .

6. Morgenstern R, Yeh R, Castano A, Maurer MS, Bokhari S. ${ }^{18}$ Fluorine sodium fluoride positron emission tomography, a potential biomarker of transthyretin cardiac amyloidosis. J Nucl Cardiol 2017. doi:10.1007/s12350-017-0799-x.

7. Gagliardi C, Tabacchi E, Bonfiglioli R, Diodato S, Nanni C, Guidalotti P, et al. Does the etiology of cardiac amyloidosis determine the myocardial uptake of [18F]-NaF PET/CT? J Nucl Cardiol 2016. doi:10.1007/s12350-016-0457-8.

8. Van Der Gucht A, Galat A, Rosso J, Guellich A, Garot J, Bodez D, et al. [18F]-NaF PET/CT imaging in cardiac amyloidosis. J Nucl Cardiol 2015;23:846-9. doi:10.1007/s12350-015-0287-0.

9. Trivieri MG, Dweck MR, Abgral R, Robson PM, Karakatsanis NA, Lala A, et al. 18F-Sodium Fluoride PET/MR for the assessment of cardiac amyloidosis. J Am Coll Cardiol 2016;68:2712-4. doi: 10.1016/j.jacc.2016.09.953. 